\title{
DNA-the phantom effect, quantum hologram and the etheric body
}

\section{Opinion}

While modern day science used to look at DNA as a material object that was fixed in nature, therefore unable to be changed, where we were at the "mercy of our genes" so to speak, we now know that this isn't true. DNA is actually composed of a liquid crystalline substance that acts as a form of antenna, receiver, and transmitter of holographic information. It's constantly in the process of taking in information from its environment and the ether as signs, archetypes, and imagery and translating it into holograms. It operates predominately out of radionics where whatever frequency its tuned to, is acts as a receiver for various forms of information within that same frequency that comes in as an acoustic wave that serves to form an electromagnetic field (EMF) as a holographic shape that's composed initially of subtle energy, which provides the blueprint or spatial mapping for constructing an exact replica as its material equivalent. Information inherent in the Ether (Akasha) always comes as a "pairing" or "wave coupling" (like the double helix) that contains both an acoustic sound and optical (visual) image as the geometric patterning inherent in the vibratory frequency.

The two waves of information form an interference pattern that together produce a 3-D holographic image as the subtle template for constructing the material body through a growth and development process. This holographic image as an invisible energy field organizes and animates matter into what's called the "phantom effect". This phantom is an invisible 3-D shape as a field formed out of information as a dynamic series of interrelated planes or parallel interlaced and correlating dimensions that operate without any cross-talk to form a chain-of-association as phase conjugation adaptive resonance. When one wave (Monad) resonates with another of the same or similar frequency, they're absorbed into each other forming an interference pattern (Dyad), where certain properties are cancelled out or contradicted, and others are matched and amplified. This adaptation process reformulates the initial generic form (archetype) into a unique variation as the couplings offspring or combination. This reformulating of internal properties to form a new whole comes by way of what we call "natural selection" as the interaction of complementary opposites that either activate or inactivate each other.

When studying DNA from a purely material perspective of constructing proteins out of encoded genetic information as the selection of qualities from each parent, it was determined that only about $2 \%$ of our DNA accounted for this process and the other $98 \%$ was what they called "junk DNA", which simply meant they didn't know what it was used for. We now know that the other $98 \%$ actually serves as a form of memory bank where information is both written or encoded, and read or decoded, to form a virtual reality out of the information as the pattern or configuration inherent in the vibratory frequency. This works in much the same way the Akashic field of Esoteric Sciences works, where information is contained within the astral plane as archetypal ideas that serve as a generic prototype for creating in the physical realm and are accessed and absorbed into the mind through sympathetic resonance. Within this same astral plane is also stored "thought-forms" produced by humans as emotional
Volume 7 Issue I - 2018

\author{
Linda Gadbois \\ Spiritual Scientist, USA
}

Correspondence: Linda Gadbois, Spiritual Scientist, Colorado Springs, CO 80915, USA, Tel 3037269344,

Email Dr.lindagadbois@outlook.com

Received: January 18, 2018 | Published: January 29, 2018

memories that exist as a holographic template that's "recorded" on the Ether (the Akashic book of Life), and not only forms the memory of our soul and body, but also populates the Astral plane of what we call "the Collective Unconscious", or mass consciousness with virtual memories. Our DNA as a crystalline transmitter and receiver, draws in (resonates with) the thought-forms of others (group mind) as well as ideas from the higher dimension of the mental plane of Universal archetypes, where both come as the holographic information that ultimately serves to program our DNA.

DNA operates by the same principles as the mind and brain, where the acoustic aspect of information as "words" acts to form a visual holographic image in the imagination that turns the idea inherent in the words into a virtual reality. It translates ideas that are communicated by talking about them, whether through our own thoughts as internal dialogue, or as listening to someone else talk, into visual imagery as living scenarios. It comes as words, sentences, paragraphs, and pages of written and spoken script that forms visual imagery in our mind's eye as we read it (absorb it). DNA works by way of the same principles as the mind and neurons of the brain and body. It decodes words into 3-D realities as the basis for organizing matter into the biological form that corresponds to the image as an archetypal idea. This is represented in Sacred Geometry by the Tetrad (Tetragrammaton), which is the physical outward reflection and projection of the Triad (inner imagining) that emerges naturally out of the Dyad, which symbolizes an interference pattern as the coupling of two wave forms (double helix) of the same frequency to produce a new whole through coherence. Most spiritual texts describe God as the creator calling forth all material life using words as breath that moves across the water (liquid crystal), causing a form to rise up and take shape.

While the genetic make-up of our body doesn't change very much throughout our lifetime, our inner constitution as our character and mental paradigm (vibratory structure) can often change quite drastically. As our inner constitution changes, how we see and what we see in the outer world changes simultaneously. This is because the inner and the outer act as two wave forms that resonate with each other forming an interference pattern that activates different aspects while deactivating others, changing how it's configured. We only see in everything else what's of the same nature (frequency) as we are. As we grow, develop into higher states, and transform mentally and emotionally, how things appear to us changes accordingly. You know when you've undergone transformation of some form by the fact that 
you begin seeing others and the world in general in a different way. What we notice and how we interpret things to give them meaning changes as an outer reflection of our inner state.

DNA, like the mind, is a fluid-like substance that's always being re-informed by an energetic exchange of subtle energy with everything else around it that's of a similar vibration causing it to constantly flux and morph. It's like a shimmering luster morphing moment by moment based on what new information or qualities it's absorbing from the environment that modifies its state. Words carried on a certain frequency are naturally inducted into the individual mind where they form an internal image as the reality indicated by the words. Whenever our mind is in a passive and receptive state, such as meditation and hypnosis (Theta-Alpha state), where there's no editing or resistance from the conscious mind (outer awareness), ideas are readily taken in as suggestion, allowed to rise up in the imagination and take hold, and entire realties as an experience are constructed out of them. These holographic realities create a form of inner experience that acts directly on the subconscious mind as the body's consciousness (DNA), to program it through virtual memories.

Matter as particles held together by an invisible electromagnetic field is what forms the primary substance of what we call reality. Matter itself doesn't "possess or generate" consciousness of its own, but acts as the passive receptor for consciousness as vibratory information that structures it into a holistic biological living system. DNA acts as the subtle antenna and receiver for acoustic information that forms a holographic image as an electromagnetic field that provides the blueprint as the etheric body used to construct the physical body. Form and properties always indicates function and how a system operates and behaves. Whenever we change the information used to structure and operate a system, we change how the system forms, expresses, and functions as a whole. The genetic code of our DNA isn't static and fixed, but rather dynamic and always in the process of transforming based on the information as language of some sorts that it acts to absorb, interpret, and shape into an idea.

Scalar energy, as subtle energy, readily moves through and into matter and divides forming an electromagnetic field that serves to organize astral light as essence (photons) into the holographic reality as one possibility (potential) inherent in the scalar wave. Scalar waves exist as a unified field of subtle energy that exists everywhere as what we call "empty space". This empty space, that's commonly called a quantum vacuum, isn't empty at all, but rather filled with holographic information as archetypes used to form, hold together, and sustains the entire material world. It's the invisible field that organizes matter into organic and inorganic biological systems that are comprised of both an active (animate) and passive (inanimate) aspects.

What this shows us is that our DNA as our subconscious mind or body consciousness is literally programmed by our own thoughts and internal dialogue that are imagined as realities, and from various forms of media that act as suggestion and become the nature of our thoughts. What we hear outwardly forms a picture of reality inwardly. This inward picture formed out of hearing something someone says, becomes a part of our thoughts and provides a holographic image that imprints the DNA of our body with that information as a form of genetic code. DNA, like the mind itself, has the ability to both write and read genetic information. Most of our thoughts are formed from what we've been taught or heard being said, that we incorporate in a harmonious fashion to form our mental paradigm as a working model for perceiving and interpreting the outer world. Any information sent on radio/microwaves in the form of language and pictures, that we take in and think about, not only becomes a part of our vibratory essence in terms of our thinking and feeling, but also as the programming for the DNA of our molecular structure as a corresponding physical equivalent, or the reality inherent in the thoughts.

As we think through a form of internal dialogue where we're talking to ourselves, or whatever we hear and listen to going on around us that we take in and actually think about, turning it into an imagined reality, we're encoding ourselves with that information as a form of hypnotic suggestion. The most important aspect of our physical development and well-being comes from our thoughts and what type of ideas we expose ourselves to and actively engage in. Anytime we're watching $\mathrm{TV}$, listening to the radio, music, talking on our cell-phone, browsing the internet reading and watching things, we're "programming ourselves" with that information. We're becoming "one with it" by creating an inner reality out of it, and we're tuning ourselves to that same vibration. What we hear and watch becomes a part of our natural thoughts. All we're ever really doing is running the same "type of ideas" through our mind over and over, changing them only through adaptive resonance as a modified application to a different or unique scenario.

The most important aspect of our personal development is controlling our own thoughts, intentionally directing our attention onto desirable and beneficial ideas, and monitoring what we expose ourselves to in terms of others and various forms or media. Whomever or whatever we associate with, we take in and become like. Spoken words, whether internally or externally, form holographic images in our mind that are impressed on the ether, and permanently recorded as a memory. Just as we live our life out of memory, thinking and dwelling in the past as a way of creating the future in the present by using the same idea to create a new variation, our body is regenerated and sustained out of the same memory. The mind forms an astral image as a hologram that imprints the etheric body with the information of that image.

The etheric body as an electromagnetic field uses that same holographic imagery to organize and animate the cellular structure of the body to form a corresponding metaphorical equivalent. The parallel planes that ultimately result in our physical body, operate as a form of "step down process" or phases of vibration becoming a material form through a chain-of-association as charged plasma, that organizes essence as a form of gas, that coagulates into a liquidlight form, and ultimately results in a solid form. The causal field of the Luminiferous Aether or Akashic Field of vibratory frequencies as archetypal information is absorbed into our mind and shaped into a personal reality that simultaneously elicits an emotional response to our own thoughts, infusing them with meaning that becomes the "motivating force" that animates them into a storyline or dialogue of some kind. This imaginary sensory reality is simultaneously received by, interpreted, and used to produce an equivalent effect in the etheric hologram of the body, reprogramming and modifying it accordingly. Our mind receives information that it turns into emotional thoughts that act as a blueprint for shaping our material body, both inwardly and outwardly. We literally become what we think and imagine.

\section{Acknowledgements}

None.

\section{Conflict of interest}

The author declares no conflict of interest. 